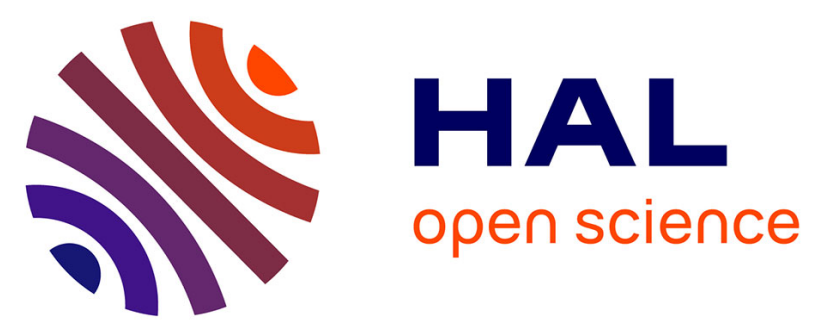

\title{
Predicting the Impact of Magnetic Components Used for EMI Suppression on the Base-Band of a Power Amplifier
}

Roberto Mrad, Gaël Pillonnet, Florent Morel, Christian Vollaire, Angelo

Nagari

\section{- To cite this version:}

Roberto Mrad, Gaël Pillonnet, Florent Morel, Christian Vollaire, Angelo Nagari. Predicting the Impact of Magnetic Components Used for EMI Suppression on the Base-Band of a Power Amplifier. IEEE Transactions on Power Electronics, 2015, 10.1109/TPEL.2014.2351421 • hal-01259800

\section{HAL Id: hal-01259800 \\ https://hal.science/hal-01259800}

Submitted on 21 Jan 2016

HAL is a multi-disciplinary open access archive for the deposit and dissemination of scientific research documents, whether they are published or not. The documents may come from teaching and research institutions in France or abroad, or from public or private research centers.
L'archive ouverte pluridisciplinaire $\mathbf{H A L}$, est destinée au dépôt et à la diffusion de documents scientifiques de niveau recherche, publiés ou non, émanant des établissements d'enseignement et de recherche français ou étrangers, des laboratoires publics ou privés. 


\title{
Predicting the Impact of Magnetic Components Used for EMI Suppression on the Base-Band of a Power Amplifier
}

\author{
Roberto Mrad, Member, IEEE, Gaël Pillonnet, Member, IEEE, Florent Morel, Member, IEEE, \\ Christian Vollaire, Member, IEEE, and Angelo Nagari, Member, IEEE
}

\begin{abstract}
Class-D audio amplifiers are switching circuits that produce serious ElectroMagnetic (EM) emissions and disturb the surrounding electronics. In order to reduce these emissions, ElectroMagnetic Compatibility (EMC) filters with ferrite beads are used. However, ferrite beads contain magnetic materials that have a nonlinear behavior. Thus, they have an unfavorable impact on the system audio quality. The common ferrite bead models do not take into account nonlinear phenomena. Thus, to predict the impact on the signal quality, this paper models the ferrite bead using the Jiles - Atherton magnetic material theory. The presented model provides the designers with a tool to quantify the effect of EMC filters on the Total Harmonic Distortion (THD) of audio amplifiers. The simulated and measured results show that the tested ferrite bead have a negative effect on the audio signal for a wide range of amplitudes and can increase the THD up to $37 d B$. Finally, this paper highlights the impact of the magnetic material type on the audio distortion by simulating the same component with different types of materials.
\end{abstract}

Index Terms-Ferrite bead, Magnetic material, Hysteresis loop, Jiles - Atherton, THD, Audio amplifier, Class-D amplifier, EMC filter.

\section{INTRODUCTION}

Often in embedded systems, batteries are the only source of energy. Thus, for such applications and for a longer lifetime battery, electronic circuits with a higher power efficiency are carefully chosen. In audio applications, Class-D audio amplifiers present the highest power efficiency compared to other amplifier types [1] and are widely used as audio drivers in battery-powered systems such as smartphones.

The Class-D amplifier has the same switching behavior as a DC-DC buck converter (Fig. 1). The input reference is an audio signal which is modulated by Pulse Width Modulation (PWM) to generate a high frequency pulse train (about a few hundreds of $\mathrm{kHz}$ ) [2]. Thus, the output spectrum contains not only the audio frequencies in the audio frequency band [20 $\mathrm{Hz}-20 \mathrm{kHz}$, but also the high frequency harmonics due to the PWM switching. Even though, high frequencies are not audible, they produce high frequency ElectroMagnetic (EM) emissions that disturb the surrounding equipments, as well as the nearby circuits on the same Printed Circuit Board (PCB). Many published works focus on reducing these emissions with a circuit solution [3]-[5]. Many other solutions have proposed filterless Class D amplifiers thanks to the speaker inductive nature [6] and its mechanical frequency response. However, the ElectroMagnetic Interference (EMI) filter is still mandatory in order to reduce the unwanted emissions and prevent any sys-

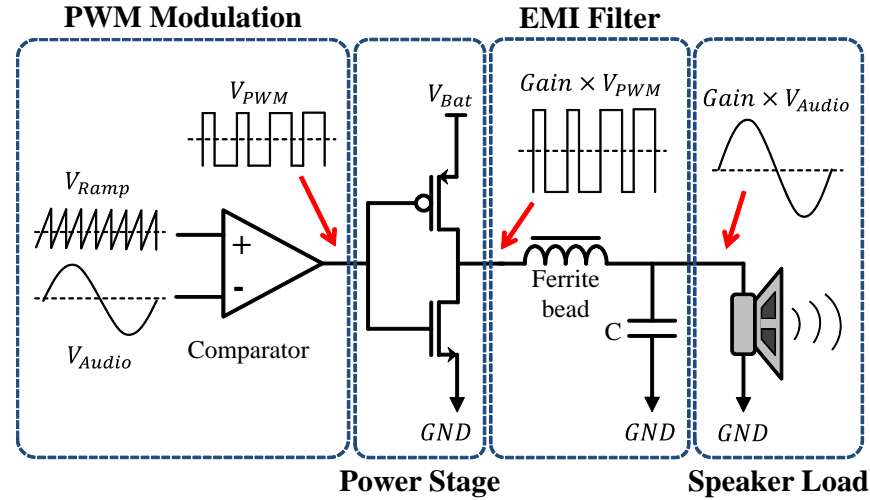

Fig. 1. Class-D amplifier architecture.
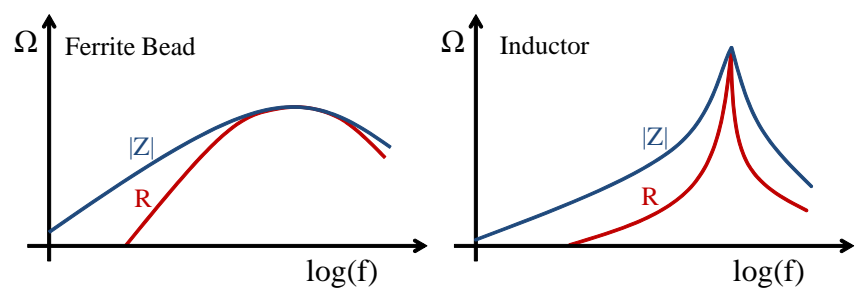

Fig. 2. Ferrite bead impedance (inspired from [7]).

tem dysfunction, especially the radio communication (GSM, FM, etc.). Technically, the EMI filter has to prevent over the entire frequency band any perturbations from propagating to the speaker while keeping the audio signal in the audio frequency band intact.

For the integrated solutions such as smartphones, SurfaceMounted Technology (SMT) ferrite beads are used due to their impedance behavior along the frequency band. They allow a broadband filtering effect compared to an inductor (Fig. 2). In addition, ferrite beads are mainly resistive at high frequencies, thereby, they can dissipate the high frequency disturbances as heat (Fig. 2). The magnetic material gives the ferrite bead a higher inductance when used in the inductive region (Fig. 2) and a higher dissipation capability when used for EMI suppression. However, due to its saturation and hysteresis characteristics, the magnetic material introduces a non-linear effect into the component behavior. Thus, when used with 
-Without ferrite bead $($ THD = -69 dB) $\square$ With ferrite bead $($ THD = -53 dB)

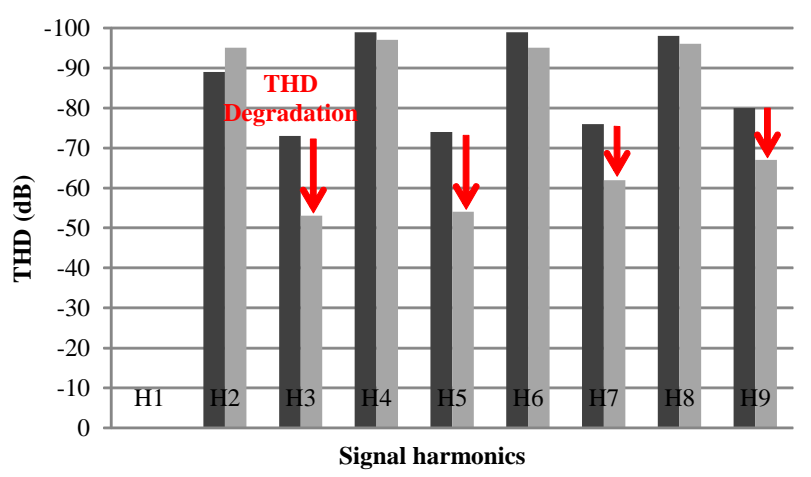

Fig. 3. Example of Class-D THD and bargraph measurement. Note that, the more the bars are high the more the voltage is small since the scale is an inverted $d B$ scale.

a Class-D amplifier, besides the EMI suppression capability, ferrite beads have a direct impact on the audio signal quality. Fig. 3 shows an example of a Total Harmonic Distortion (THD) measurements for a Class-D amplifier with and without ferrite beads, for an output signal having a frequency of $1 \mathrm{kHz}$, an output power of $100 \mathrm{~mW}$ and an $8 \Omega$ speaker load. Knowing that the ferrite bead used was chosen for its low THD degradation, it increases the THD from $-69 d B$ to $-53 d B$ which is a significant audio quality degradation. Moreover, Fig. 3 shows an increase in the odd harmonics and slight variation in the even harmonics. Note that the THD is the ratio of the sum of power of all the harmonics in the audio band over the fundamental frequency power.

In previous work [8], [9], the audio distortion caused by intermodulation or by switching transitions has been studied. However, the effect of the EMI filter was not been taken into account. In [10], measurements were done on a ferrite bead to experimentally quantify its effect in the audio frequency band. The results show a negative impact on the audio signal which can be seen by an increase in the THD, for the entire audio frequency range and for a wide range of amplitudes. However, by the authors best knowledge, there is no publication in the literature that simulate this phenomenon in the audio field. The most common model for ferrite beads is a linear model constructed by impedance matching [11], [12]. It is used for EMI simulations but is not able to predict the audio quality. Many other papers deal with modeling the magnetic materials for power electronics issues, such as inductors [13], [14], transformers [15], [16] or electric machines [17], [18]. To study its impact on the audio signal, this paper models the ferrite bead using the Jiles - Atherton theory [19]. It models the impact on the output voltage with a $1 \mathrm{kHz}$ sinusoidal current and explains the occurring phenomenon. In addition, the THD is calculated and compared to the THD of the measured signals at different amplitude levels.

The paper is organized as follows. It starts by modeling the ferrite bead in section II. Thus, an overview on the magnetic material modeling is presented. Then, it includes the physical architecture of the component. It proceeds to validate the model by comparing the simulation to the measurement in section III. This shows the time domain and the frequency domain results, as well as the THD for different amplitude levels. Section IV studies the influence of the magnetic material type on the audio distortion. Finally, section V summarizes and concludes the paper.

\section{FERRITE BEAD MODELING}

Modeling a ferrite bead can be separated in two parts: magnetic material modeling and physical architecture modeling. Both are discussed in this section and a simulation is shown in order to explain the occurring phenomenon.

The most common magnetic material models including the hysteresis and saturation are :

- D. C. Jiles and D. L. Atherton model [19]-[21], known as the Jiles - Atherton (JA) model

- John Chan et al. model [22], known as the Chan et al. model

- Ferenc (Franz) Preisach model [23]-[25], known as the Preisach model

- C. D. Boley and M. L. Hodgdon [26], known as the Hodgdon model

In the present work, the JA and the Chan et al. models have been used because they are accurate, practical to implement and widely used for ferrite materials [27]. The results from both are very similar, however, only the results of the JA model are presented here. The reason is because we have access on JA parameters for different magnetic materials and the parameters of the Chan et al. model were deduced from the JA model simulating a given magnetic material. Thus, only an overview on the JA model is explained in this paper and a possible implementations can be found in [28].

\section{A. Jiles - Atherton model}

The JA model, in contrary to many other models in the literature, is based on a physical approach to describe the hysteresis loop [19]. The main idea behind it is to consider the energy related to the wall (known as Bloch wall) movements of the magnetic domains (known as Weiss Domains) inside a magnetic material. The hysteresis phenomenon is described as a friction force due to the wall movement during the magnetization process [28].

The JA model is related to two mechanisms. The first is the irreversible domain wall motion represented by $M_{i r r}[A / m]$. The second is reversible domain wall bending represented by $M_{\text {rev }}[A / m]$. The magnetization $M[A / m]$ of the magnetic material can thus be given by (1).

$$
M=M_{i r r}+M_{r e v}
$$

$M_{i r r}$ and $M_{r e v}$ are described by (2) and (3), respectively.

$$
\begin{aligned}
\frac{\mathrm{d} M_{i r r}}{\mathrm{~d} H_{e}} & =\frac{\left(M_{a n}-M_{i r r}\right)}{k \delta} \\
M_{r e v} & =c\left(M_{a n}-M_{i r r}\right)
\end{aligned}
$$

where, $M_{a n}[A / m]$ is the anhysteretic magnetization given by (4). $H_{e}[A / m]$ is the effective magnetic field given by (5). $k[A / m]$ is a parameter linked to the coercive field. If 


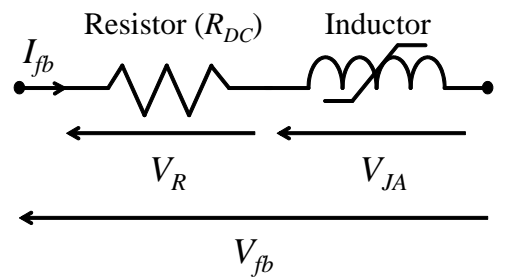

Fig. 4. Ferrite bead model in the audio frequency band.

$H[A / m]$ is the external magnetic field and $t[s]$ is the time, $\delta$ [dimensionless] would be equal to +1 if $\frac{\mathrm{d} H}{\mathrm{~d} t}>0$ and -1 if $\frac{\mathrm{d} H}{\mathrm{~d} t}<0$. Finally $c$ [dimensionless] is the reversible coefficient.

$$
\begin{gathered}
M_{a n}\left(H_{e}\right)=M_{\text {sat }}\left[\operatorname{coth}\left(\frac{H_{e}}{a}\right)-\left(\frac{a}{H_{e}}\right)\right] \\
H_{e}=H+\alpha M_{a n}
\end{gathered}
$$

with $M_{\text {sat }}[\mathrm{A} / \mathrm{m}]$ is the saturation magnetization, $a[\mathrm{~A} / \mathrm{m}]$ is the shape parameter of $M_{a n}$ and $\alpha$ [dimensionless] is the domain interaction parameter.

Thus, (1) to (5) allow us to deduce the JA equation (6) that gives the variation of the magnetization $M$ in terms of the magnetic field $H$.

$$
\frac{\mathrm{d} M}{\mathrm{~d} H}=\frac{(1-c) \frac{\mathrm{d} M_{i r r}}{\mathrm{~d} H_{e}}+c \frac{\mathrm{d} M_{a n}}{\mathrm{~d} H_{e}}}{1-\alpha c \frac{\mathrm{d} M_{a n}}{\mathrm{~d} H_{e}}-\alpha(1-c) \frac{\mathrm{d} M_{i r r}}{\mathrm{~d} H_{e}}}
$$

Finally, the magnetic induction $B[T]$ can be related to the magnetization $M$ and the magnetic field $H$ by (7)

$$
B=\mu_{0}(H+M)
$$

where $\mu_{0}=4 \pi e^{-7}[H / m]$ is the magnetic permeability of the vacuum.

Each magnetic material has its own hysteresis characteristics. Thus, JA allows us to configure the model for a given magnetic material using the 5 parameters $\alpha, a, k, c$ and $M_{\text {sat }}$. The determination of these parameters has been widely studied and discussed [29]-[31]. Table I gives some examples for 3 different magnetic materials [28].

TABLE I

JA PARAMETERS FOR 3 DIFFERENT MAGNETIC MATERIALS [28]

\begin{tabular}{lccc}
\hline Parameter & Ferrite N30 & FeSi & Iron powder \\
\hline$\alpha$ dimensionless] & $9.77 e^{-5}$ & $1.31 e^{-4}$ & $1.83 e^{-3}$ \\
$a[A / m]$ & 20.25 & 59.5 & 1642 \\
$k[A / m]$ & 55.75 & 99.2 & 1865 \\
$c$ [dimensionless $]$ & 0.9 & 0.54 & 0.8 \\
$M_{\text {sat }}[A / m]$ & $28.2 e^{4}$ & $11.5 e^{5}$ & $11.2 e^{5}$ \\
\hline
\end{tabular}

\section{B. Physical architecture modeling}

The ferrite bead is an electric dipole. In the audio frequency band, it can be modeled as shown in Fig. 4. The resistor $R_{D C}$ is the DC resistor of the component. The ferrite bead voltage can be then described by (8)

$$
V_{f b}(t)=V_{J A}(t)+R_{D C} \cdot I_{f b}(t)
$$

where the voltage $V_{J A}(t)$ is the inductive part of the component and can be related to the magnetic induction $B(t)$ using (9). $I_{f b}(t)$ is the component current and can be related to the magnetic field $H(t)$ using (10).

$$
\begin{gathered}
V_{J A}(t)=N \cdot S \cdot \frac{\mathrm{d} B(t)}{\mathrm{d} t} \\
I_{f b}(t)=\frac{L}{N} \cdot H(t)
\end{gathered}
$$

where $N$ is the coil number, $S\left[\mathrm{~m}^{2}\right]$ is the component effective section, $L[m]$ is the effective component length, and $t$ is the time.

Finally, the relation between $B(t)$ and $H(t)$ given by the JA model, allows us to have the relation between $V_{J A}(t)$ and $I_{f b}(t)$.

This model is implemented and simulated in MATLAB environment. In order to expose the occurring phenomenon, a simulation example for a sinusoidal current of $22 \mathrm{~mA}$ and for a Ferrite N30 material is presented. Note that the model parameters are presented in Table II and the procedure of parameter extraction is presented in the next section. The hysteresis loop is shown in Fig. 5 and the voltage results are shown in Fig. 6.

As can be seen from Fig. 6, for a sinusoidal current, the product $R_{D C} \times I$ keeps the sinusoidal form due to its linearity. However, the hysteresis magnetic loop modeled by the JA model, generates peaks in the voltage $V_{J A}\left(P_{1}\right.$ and $P_{2}$ in Fig. 6). These are caused by the high slopes of the hysteresis loop which cause a fast variation in $V_{J A}$. The peaks occur at the current zero crossing (the same as $H$ zero crossing according to equation (10)). Therefore, according to equation (8) the ferrite bead voltage is the sum of the two voltages $V_{J A}$ and $R_{D C} \times I$. As a result, the ferrite bead voltage is a non-sinusoidal signal containing peaks at the zero crossing of the waveform. The next section shows that this distortion occurs in the audio frequency band and causes audio degradation.

\section{MEASUREMENTS AND VALIDATION}

\section{A. Measurements}

The ferrite bead cited in [32] has been used for measurements in order to validate the proposed model. This component was chosen because it has a high internal DC resistance $R_{D C}$ (maximum of $1.5 \Omega$ ) even though it has a low nominal current $(50 \mathrm{~mA})$. This allows us to have a high voltage across the ferrite bead, allowing a better Signal to Noise Ratio (SNR) while measuring this voltage $\left(V_{f b}\right)$. Notice that in industrial Class-D applications, ferrite beads are chosen with a lower internal DC resistance (around $50 \mathrm{~m} \Omega$ for mobile phones) for better efficiency and a higher rated current (around $2 A$ for mobile phones) for thermal reasons.

The chosen ferrite bead is mounted on a PCB with an $8 \Omega$ load similar to a speaker. The schematic is shown in Fig. 7 and used to validate the ferrite bead model, also, to evaluate the impact of the chosen bead on the audio distortion. Replacing the loudspeaker by a resistor will change the impedance behavior of the load. However, these changes do not impact the 


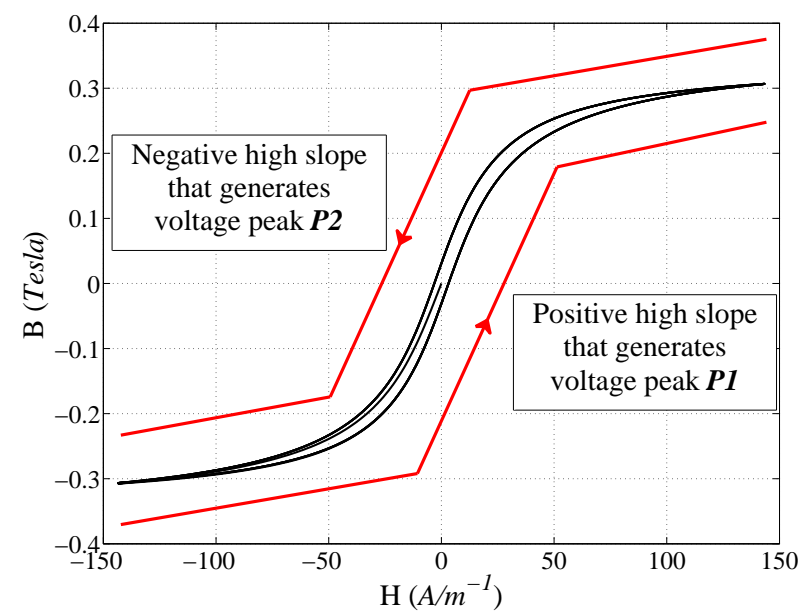

Fig. 5. Hysteresis loop obtained by the JA model and the parameters of the first column in Table I.

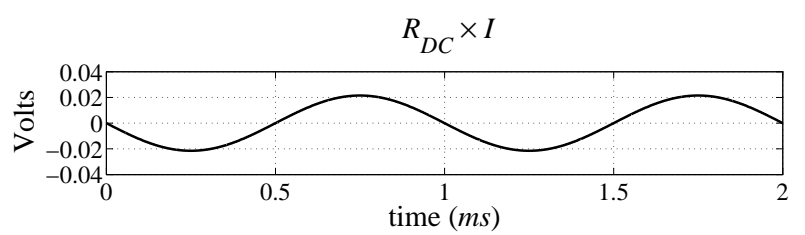

$V_{J A}$

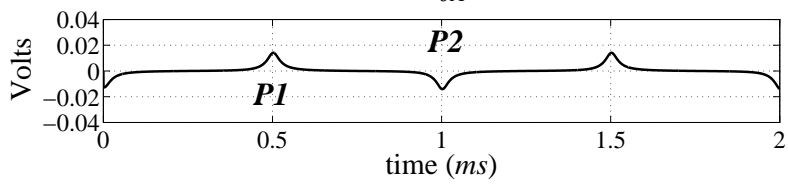

$V_{f b}=V_{J A}+R_{D C} \cdot I$

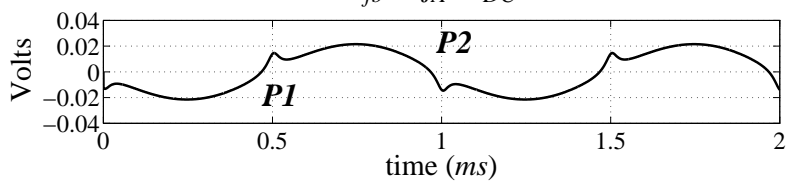

Fig. 6. A simulation example showing the occurring phenomenon.

distortion of the electric audio signals. The nonlinear behavior of the loudspeaker and the capacitor are negligible compared to the one of the ferrite bead. This does not change then the analysis on a sinusoidal waveform because the current keeps the sinusoidal shape. The circuit is powered by a linear amplifier instead of a Class-D one. The main reason for this is to remove the high frequencies of a PWM spectrum and study the impact of the ferrite bead only in the audio frequency band. The signal generator delivers burst pulses of 5 sinusoidal periods with a frequency of $1 \mathrm{kHz}$. The pulses are separated by a delay of one second to avoid heating the component which would change the magnetic material characteristics. For $V_{i n}=1 \mathrm{~V}$, the results of the ferrite bead voltage $V_{f b}$ and current $I$ are shown in Fig. 8. It shows a similar voltage behavior as the one obtained by simulation in Fig. 6. This proves that using this model is appropriate for these ferrite beads. Peaks appear at the zero crossing of the signals (around $0 \mathrm{~s}, 0.5 \mathrm{~ms}, 1 \mathrm{~ms}$ and $1.5 \mathrm{~ms}$ ). However, slight variations are

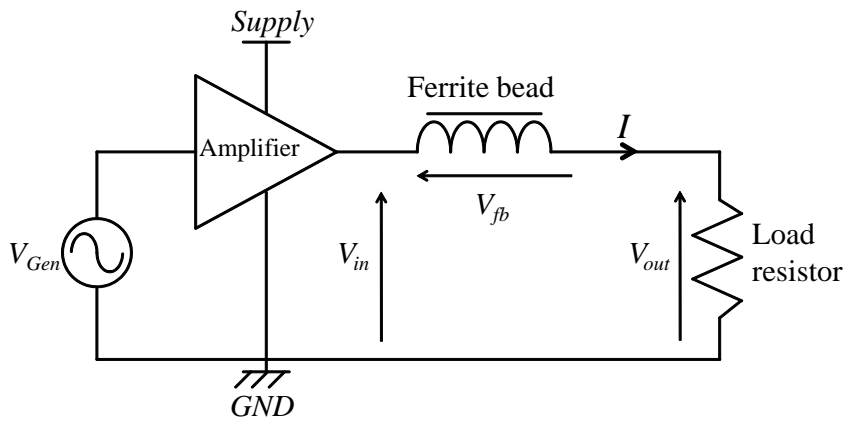

Fig. 7. Measurement schematic.
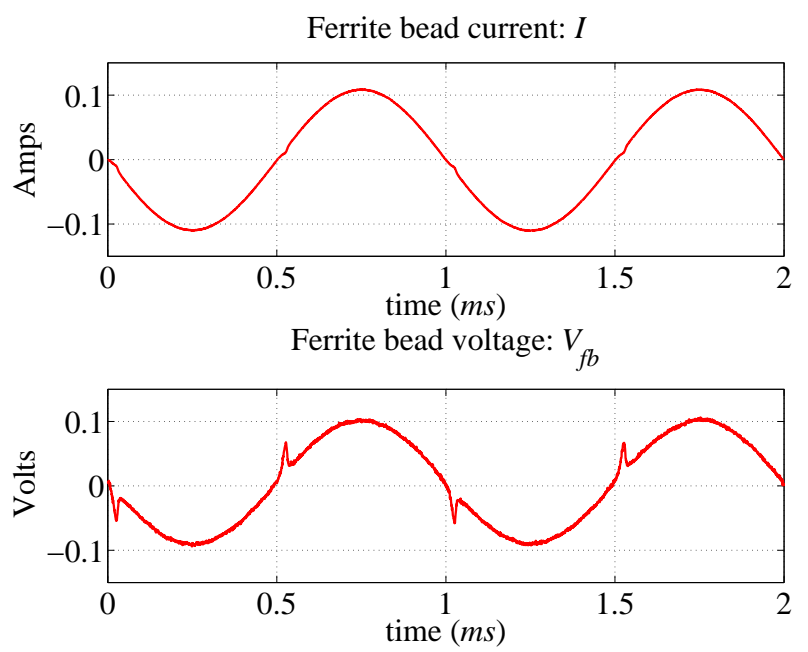

Fig. 8. Measurement results.

also seen in the ferrite bead current at the same time as the voltage peaks.

\section{B. Component modeling}

To model the ferrite bead [32] used for measurements, the following characteristics are needed: the DC resistance $R_{D C}$, coil number $N$, effective section $S$, effective length and the 5 parameters of the JA model that characterize the magnetic material. Nevertheless, this information is not given by the component supplier. Therefore, they are obtained as follows.

First, the DC resistance has been measured by an impedance analyzer, thus, $R_{D C}=1 \Omega$.

A ferrite bead is a coil developed inside a magnetic material as shown in Fig. 9. Thus, the component has been broken in two pieces and seen through a microscope. The picture is shown in Fig. 10, and we see that this ferrite bead has the same physical architecture as the right hand architecture in Fig. 9. The coil number is $N=9$ and the coil conductor is a pack of 3 conductors in parallel. It can be seen as well that the effective length is almost that of the component itself and the effective width is almost half that of the component width. Therefore, as the chosen ferrite bead [32] is a 0603 (1608 in metric dimension) SMT package, then according to the data-sheet 


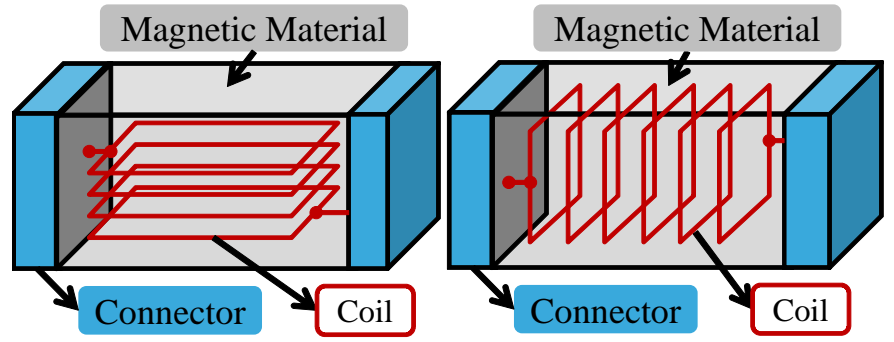

Fig. 9. Ferrite bead physical architectures.

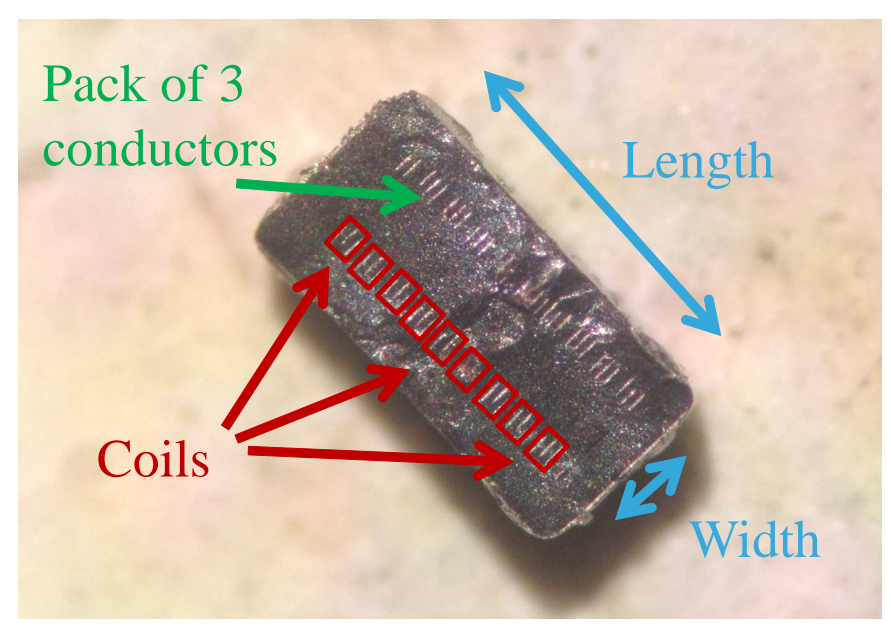

Fig. 10. Ferrite bead broken in two part.

$L$ and $S$ are $1.6 \mathrm{~mm}$ and $0.4 \times 0.4 \mathrm{~mm}^{2}$, respectively. Note that, the effective section is considered as a square section.

The ferrite bead magnetic material reference and characteristics are not given by the supplier. Thus, the 5 parameters of the JA model, as well as the 3 parameters of the Chan et al. model [22] are unknown. As the material of the beads is ferrite, then to reduce the error due to the hysteresis loop characterization, the five JA parameters were assumed to be the same as those of known ferrite material found in [28] and presented in the first column of Table I. In conclusion, the required characteristics and parameters for the ferrite bead modeling are summarized in Table II.

TABLE II

PARAMETERS FOR THE FERRITE BEAD UNDER TEST

\begin{tabular}{|ll|ll|}
\hline Parameter & Value & Parameter & Value \\
\hline$\alpha$ & $9.77 e^{-5}$ & $N$ & 9 \\
$a$ & $20.25 \mathrm{~A} / \mathrm{m}$ & $L$ & $1.6 \mathrm{~mm}$ \\
$k$ & $55.75 \mathrm{~A} / \mathrm{m}$ & $S$ & $0.16 \mathrm{~mm}^{2}$ \\
$c$ & 0.9 & $R_{D C}$ & $1 \Omega$ \\
$M_{\text {sat }}$ & $28.2 e^{4} \mathrm{~A} / \mathrm{m}$ & & \\
\hline
\end{tabular}

\section{Time domain simulations}

The specified model is now used for simulation in MATLAB. The ferrite bead and load are in series connected (see Fig. 7). The load has a higher impedance in the audio band and it is a linear component. As the current is imposed
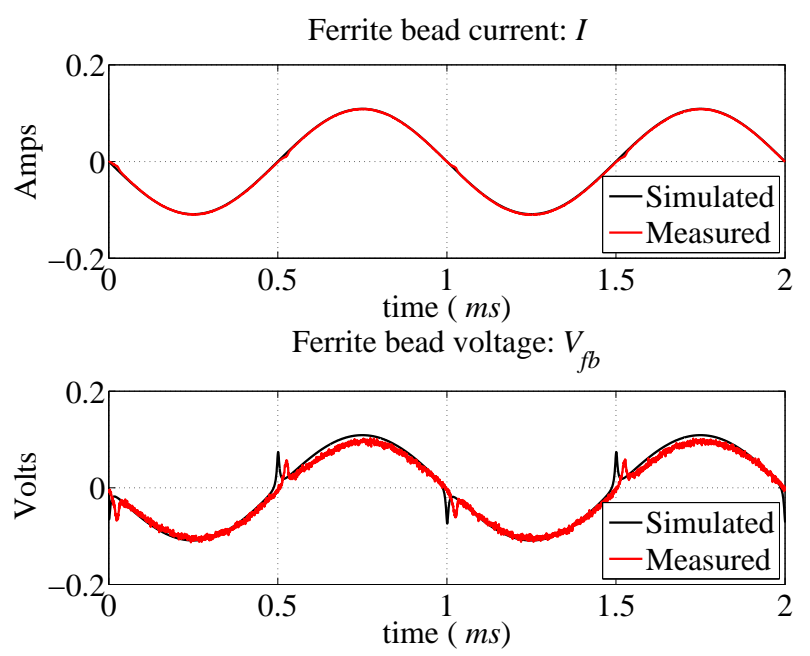

Fig. 11. Ferrite bead current and voltage.

by the higher impedance which is the load, it can thus be considered as a perfect sinusoidal waveform. This can also be seen in Fig. 8 where the current has a slight variation on the zero crossings. This assumption can be particularly valid in industrial applications as the ferrite beads are chosen with lower DC resistance $\left(R_{D C} \ll R_{L o a d}\right)$. The ferrite bead model is fed by a sinusoidal current with an amplitude given by (11)

$$
I_{\text {Peak }}=\frac{V_{\text {in }}}{R_{D C}+R_{\text {Load }}}
$$

where $V_{\text {in }}=1 \mathrm{~V}$ similar to the measurements, $R_{\text {Load }}=8.1 \Omega$ is the measured load resistance and $R_{D C}=1 \Omega$. Thus, the amplitude of the simulation current is $0.11 \mathrm{~A}$. The latter is higher than the maximum current given in the datasheet which is based on the thermal characteristics of the components. However, as the measurements were made using burst sinusoidal pulses, the maximal current can exceed the rated current (defined for steady state). In addition, at this current level the magnetic material is saturated, but in fact, at current levels lower than the component rated current, the material would also be saturated (saturation appears for currents higher than $20 \mathrm{~mA}$ ). Note also that in real audio signals, the crest factor (crest factor $=$ peak value/rms value) is generally higher than the one of a sinusoidal signal [33]. The peak value is then considerably higher than the rms current. Therefore, a current level of $0.11 \mathrm{~A}$ can be a normal operating condition when using burst pulses. Therefore, this sinusoidal current feeds the model and generates the ferrite bead voltage which is compared to the measurement in Fig. 11. As a result, the simulations are in good agreement with the measurements. However, small differences are observed in the voltage curves. The reasons for this could be the measurement uncertainty or the JA parameters uncertainty which does not refer to the actual magnetic material.

From Fig. 7, the output voltage can be given by (12)

$$
V_{\text {out }}=V_{\text {in }}-V_{f b}
$$

Thus, the output voltage across the load contains variations at 


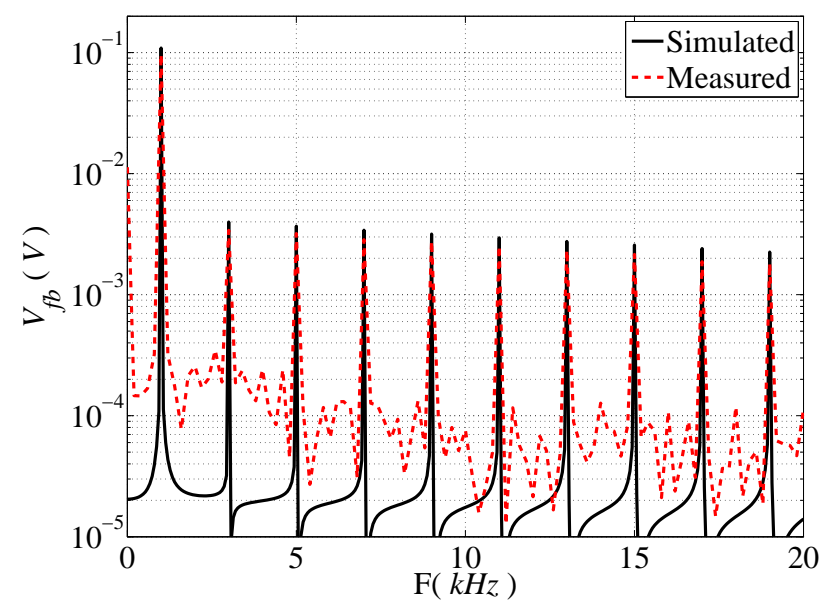

Fig. 12. Measured and simulated ferrite bead voltage.

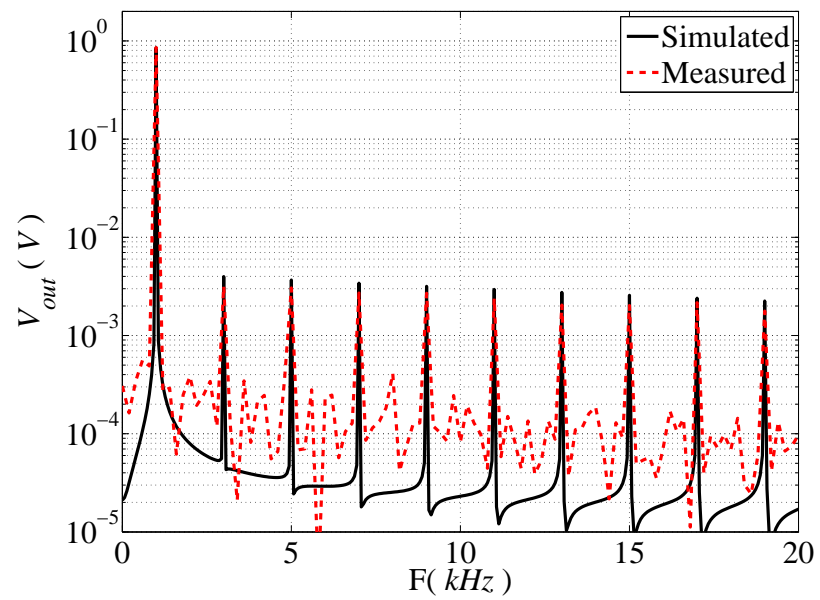

Fig. 13. Measured and simulated output voltage.

the zero crossings due to the peaks in the ferrite voltage. As the variations are small and not very visible, the time domain signals are not shown for the sake of simplification.

\section{Frequency domain comparison}

A Fast Fourier Transform (FFT) is applied to the time domain signals. The frequency domain results are shown in Fig. 12 and Fig. 13. Fig. 12 shows that the simulated ferrite bead voltage $V_{f b}$ matches the measured one in the frequency domain. It therefore allows an accurate computation of the output voltage $V_{\text {out }}$ which is shown in Fig. 13. We can see that the fundamental signal is located at $1 \mathrm{kHz}$ and the harmonics are spread over the audio frequency band. Moreover, there is also an increase in the odd harmonics of the output signal. This agrees with the case of a Class-D amplifier which is shown in Fig. 3. This can be explained by the fact that the hysteresis loop is symmetric around zero [34]. From these results, we can deduce that the ferrite bead nonlinear behavior, due to the magnetic material, is a significant source of audio degradation in the Class-D amplification system.

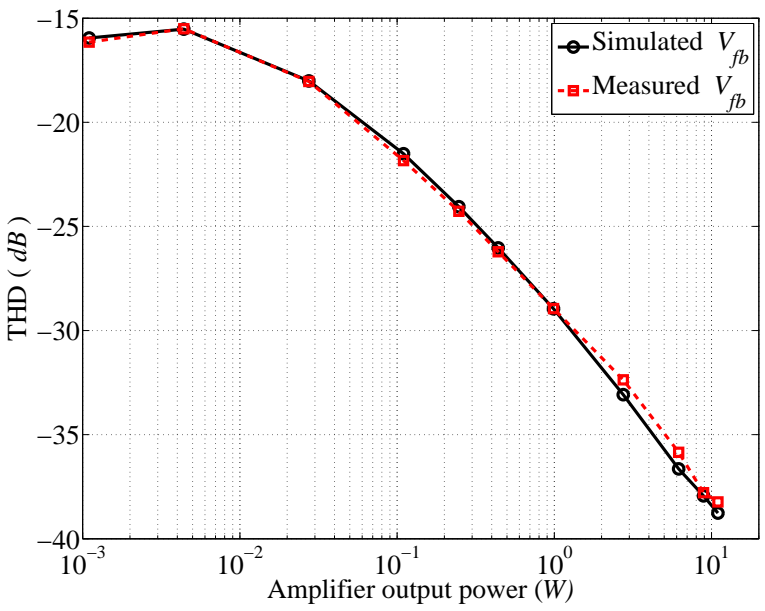

Fig. 14. $\quad V_{f b}$ THD over the amplitude.

\section{E. THD comparison}

The THD is applied in order to characterize the linearity of the audio systems. Using the spectra presented in the previous subsection, it is possible to deduce the THD of each signal. Therefore, it is calculated on each amplitude level and compared to the measured THD.

The measurements performed on the circuit in Fig. 7 have been repeated, but with different $V_{i n}$ amplitude levels. Eleven voltage levels were chosen in the range of $[100 \mathrm{mV} ; 10 \mathrm{~V}]$ which correspond to the current levels in the range of $[10.98 \mathrm{~mA} ; 1.01 \mathrm{~A}]$ and to the amplifier output power in the range of $[1.1 \mathrm{~mW} ; 11 \mathrm{~W}]$. No points have been chosen below $100 \mathrm{mV}$ due to the low measurement SNR of $V_{f b}$. Hence, the THD has been calculated for $V_{f b}, V_{\text {out }}$ and $I$ at all the amplitudes, including the measured and simulated waveforms. In addition, the THD has been measured at $V_{\text {in }}$ and $V_{\text {out }}$ using an Audio Analyzer (AA) in order to validate the THD calculation procedure. The THD plots over the amplifier output power are shown in Figs. 14, 15 and 16. Note that, the THDs for the simulated $V_{i n}$ and the simulated $I$ are not shown because they are assumed to be perfect sinusoidal waves, thus, their THD in the $d B$ scale tends toward $-\infty$.

Fig. 14 shows that the simulated THD of $V_{f b}$ matches with the measured one at all the measuring points. Note that, the audio linearity is quantified by the output voltage THD and not the ferrite bead THD. However, the latter demonstrates that the ferrite bead model is accurate over a wide range of amplitudes. Moreover, a higher impact on the signal linearity can be seen in low signal levels than in high signal levels. This can be explained as follows: in $V_{f b}$, the linear part $R_{D C} \times I$ is greater at high signal levels than the nonlinear part $V_{J A}$, the signal has therefore a better linearity at high signal levels.

From Fig. 15, three conclusions can be made. First, the simulated THD of $V_{\text {out }}$ is in a good agreement with both the THD measured using an AA and the THD calculated using the time domain measurements. Second, it shows an increase in the THD of $V_{\text {out }}$ compared with the THD of $V_{\text {in }}$, which demonstrates that this ferrite bead is able to deteriorate the 


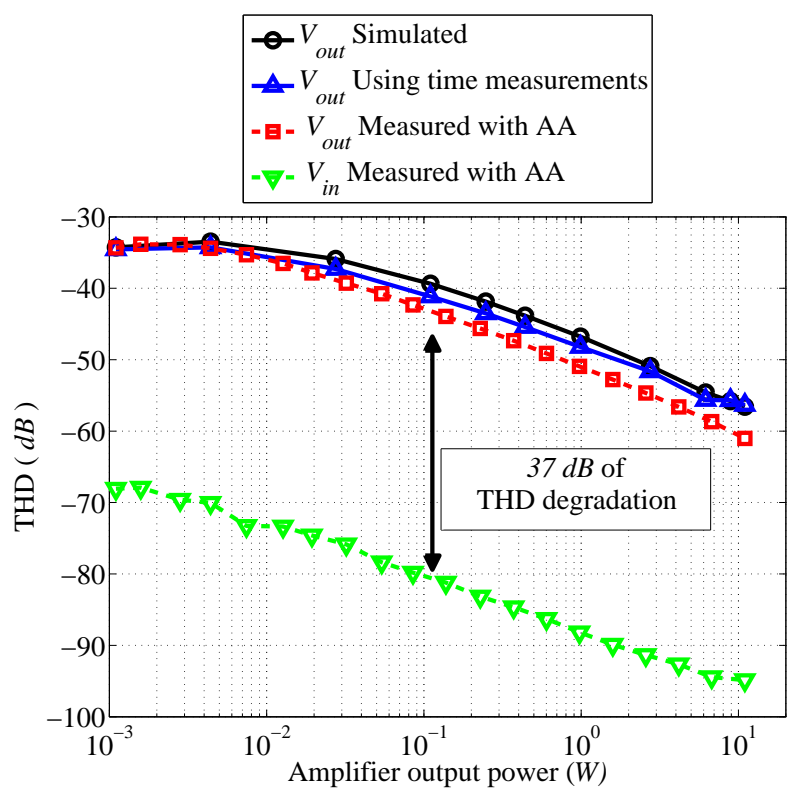

Fig. 15. $\quad V_{\text {in }}$ and $V_{\text {out }}$ THD over the amplitude.

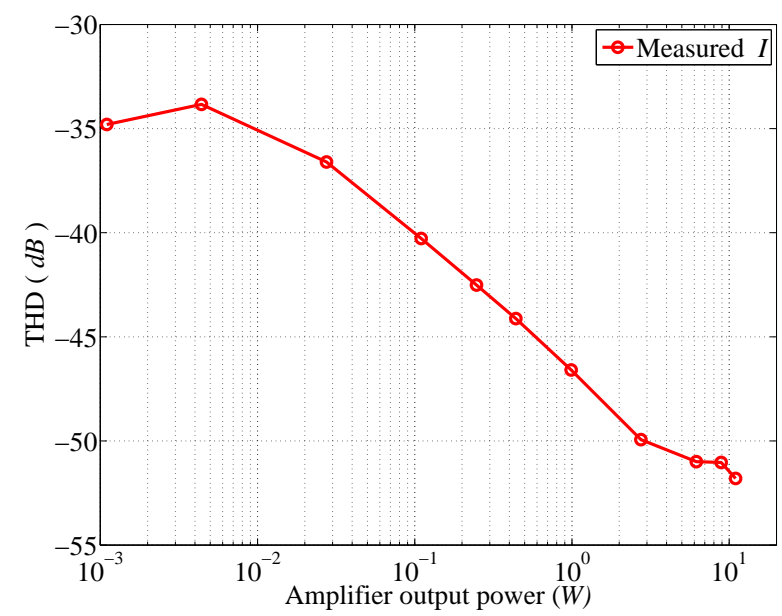

Fig. 16. The current $I$ THD over the amplitude.

signal linearity up to $37 d B$ for the amplifier in use. Such degradation is significant on the commercial level, however, further psycho-acoustic investigation is required to evaluate the impact on the human ear. Third, even if the current is lower than the ferrite bead nominal current given by the supplier (which is $50 \mathrm{~mA}$ in this case), the nonlinear behavior of the magnetic materials still have a negative impact on the output audio signal.

Fig. 16 shows that the measured current has a noticeable THD which is not as visible in time domain signals. Thus, if a high accuracy is needed from this simulation, the current should not be considered as a perfect sinusoidal waveform. However, this assumption can still be valid if the amplifier is a current source instead of voltage one [35].

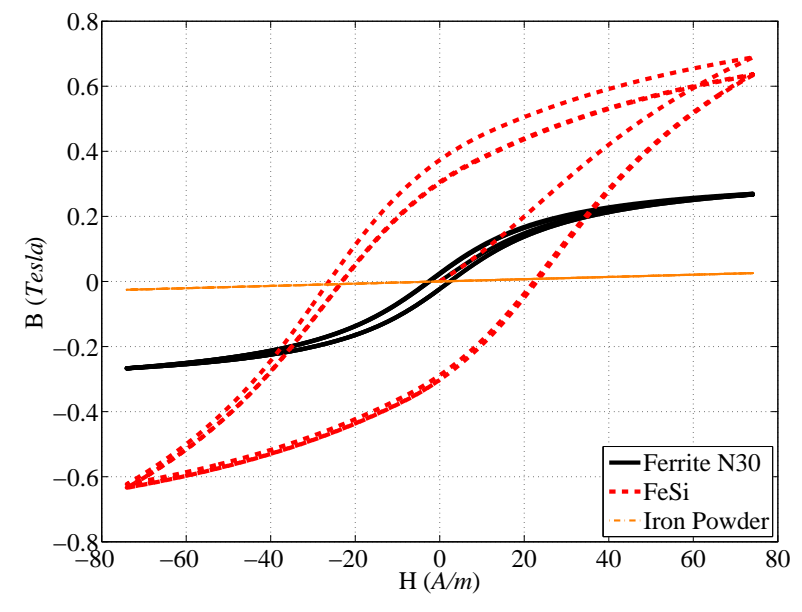

Fig. 17. Hysteresis loops obtained by the JA model, the parameters of Table I and an $11.1 \mathrm{~mA}$ peak current.

As the measurements in this paper have been made using a linear amplifier, it proves that the reason for audio degradation is mainly a low frequency phenomenon occurring in the audio frequency band.

In the integrated solutions, Class-D amplifiers are mostly differential systems. Their power stage is made by two switching cells that control the speaker. These generate opposite voltages which create the output audio signal across the speaker load. For such amplifiers, two ferrite beads are needed in the EMI filter. Thus, for the same output audio voltage, the amplifier can have double the effect observed in the case of a single ended amplifier.

\section{INFLUENCE OF MAGNETIC MATERIAL ON AUDIO DISTORTION}

Now that the model has been validated, it can be used for further simulations. What would be the difference on the signal distortion if we change the magnetic material type? This section deals with this issue and answers this question by the following.

We considered the same ferrite bead [32] and schematic (Fig. 7) that we used in the previous sections. Afterwards, for a fixed voltage level on $V_{i n}$ equal to $100 \mathrm{mV}$ (which means a current of $11.1 \mathrm{~mA}$ ), we changed the five parameters of the JA model in order to change the material type. Thus, we used the JA parameters of the "Ferrite N30", "Ferrosilicon (Fe-Si)" and "Iron Powder" materials which are available in Table I. Fig. 17 compares the different hysteresis loops obtained by the different magnetic materials. We can see that for a magnetic field of $70 \mathrm{~A} / \mathrm{m}$ (the bead current is equal to $11.1 \mathrm{~mA}$ ), the Ferrite N30 would be close to the saturation with $B=0.268 T$ (at the saturation $B \approx 0.3 T$ ), the $\mathrm{FeSi}$ would not be saturated with $B=0.63 T$ (at the saturation $B \approx 1.3 T$ ) and the Iron Powder would not be saturated and is at low magnetization levels with $B=0.025 T$ (at the saturation $B \approx 1.2 T$ ). These hysteresis loops generate the $V_{J A}$ voltages presented in the first column of plots in Fig. 18, thus, the bead voltages 

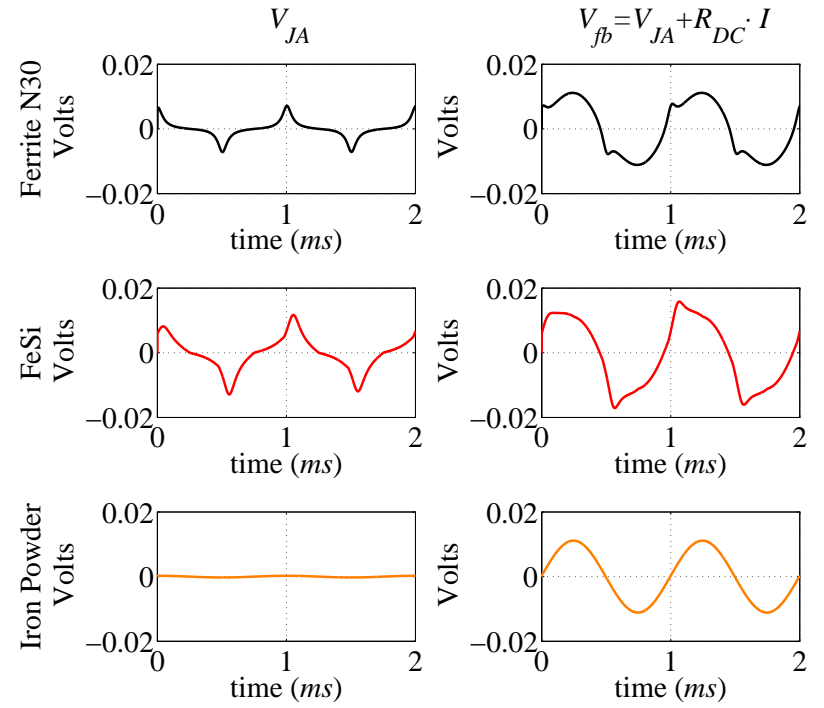

Fig. 18. Bead simulations with the three materials of Table I and a peak current of $11.1 \mathrm{~mA}$ ( $1 \mathrm{~mW}$ of audio power on the load).

presented in the second column of plots are obtained. As can be seen, the bead voltages are dissimilar if we change the type of the magnetic material. For an $11.1 \mathrm{~mA}$ current, no peaks are observed at the zero crossing of the bead voltage when using the Iron Powder material, however, the peaks are observed in the case of the two other materials. Also, in the FeSi case, the peaks are larger which must be due to the wider hysteresis loop (larger coercivity $H_{c}$ and larger remanence $B_{r}$ ) compared to the one of the Ferrite N30. Indeed, in the frequency domain the harmonic amplitudes are then different which can be seen in Fig. 19. In the case of Iron Powder, very low levels of harmonics are obtained. However, the harmonics are significantly increased when using the other two materials, knowing that, the FeSi has an unsaturated wide hysteresis loop and the Ferrite N30 has a saturated narrow hysteresis loop. Thus, not only the saturation of the magnetic material causes audio distortion, but also the hysteresis loop. Also, using the magnetic material at low levels of magnetization has a low impact on the audio distortion because the nonlinearities are not present yet in the component behavior. Moreover, it can be observed that in the case of the FeSi materials, the first harmonics are higher than those of the Ferrite N30 and the rest are lower. Thus, we can deduce that different magnetic materials can have different influences on the signal distortion.

A sweep of the voltage $V_{i n}$ has been made in the voltage range $[10 \mathrm{mV} ; 10 \mathrm{~V}]$ which corresponds to a current range of $[1.1 \mathrm{~mA} ; 1.1 \mathrm{~A}]$ and power range of $[9.9 \mu \mathrm{W} ; 9.9 \mathrm{~W}]$. The THD for these amplitudes has been computed and it is shown in Fig. 20. We can observe that the shape and level of the THD curve strongly depends on the magnetic material type. In addition, when using the FeSi or the Iron Powder materials, the THD curves have a big dissimilarity compared to the one of the Ferrite N30 which is the one that agrees with the measurement as shown in the previous

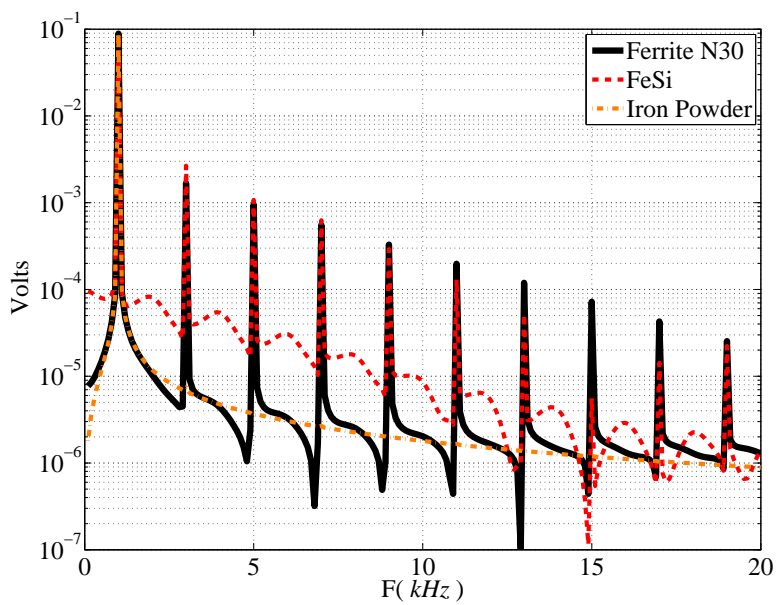

Fig. 19. Frequency domain output voltages with the three materials of Table I and a peak current of $11.1 \mathrm{~mA}$ ( $1 \mathrm{~mW}$ of audio power on the load).

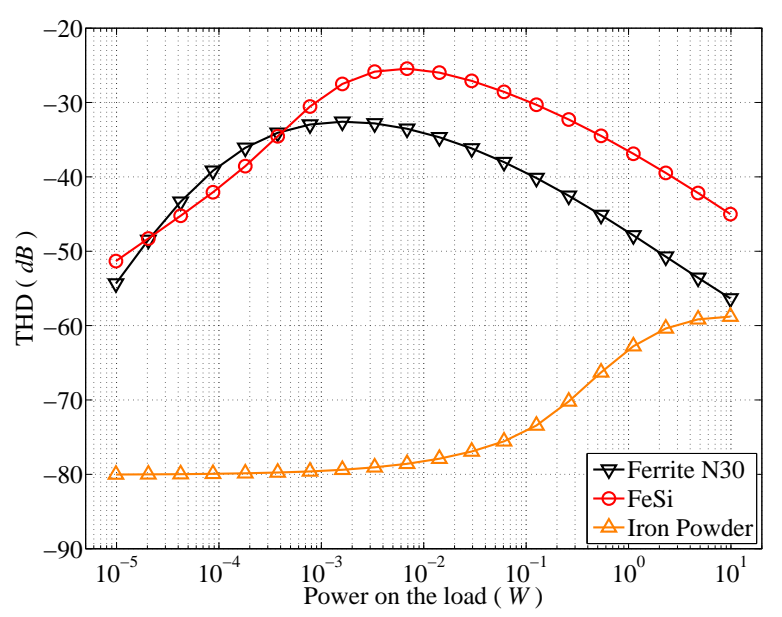

Fig. 20. THD of $V_{\text {out }}$ for different magnetic materials.

section. Therefore, we deduce that an accurate prediction of the audio distortion requires an accurate description of the magnetic material which has been made by JA modeling in this paper. Note that, a comparison between different materials from the ferrite family would be more interesting in order to improve ferrite beads for audio applications.

\section{CONCLUSION}

To conclude, using the Jiles - Atherton model for magnetic materials and the component physical architecture, a ferrite bead model has been established. It allows us to simulate these components as well as understand the occurring phenomenon when used in audio applications. This model has been compared to a measured $1 \mathrm{kHz}$ sinusoidal signal for validation. The time domain results show the appearance of voltage peaks at the zero crossing of the ferrite bead current and the frequency domain results show an increase of the signal harmonics all over the audio frequency band. We also observe an increase is the odd harmonics which agrees with 
the measurement on a Class-D amplifier. The THD has been calculated for the simulated and measured signals. A comparison with the THD measurements using an AA, shows good agreement over a wide range of amplitudes. The ferrite bead in use can generate up to $37 d B$ of THD degradation in the audio signal. Also, it has been shown that both nonlinearities, the hysteresis and the saturation can generate audio distortion and the choice of magnetic material has a significant impact on the signal distortion.

Thanks to this model, the audio system integrators are able to include the audio quality as a new criterion in the procedure of EMI filter design for switching amplifiers.

Future work will focus on implementing a dynamic magnetic material model [36], [37], in order to take into account the frequency and temperature variations that affect the ferrite bead nonlinear behavior.

\section{ACKNOWLEDGMENT}

The authors gratefully thank Alaa Hillal and Fabien Sixdenier from the Ampere laboratory, Lyon, for the valuable discussion on magnetic material modeling.

\section{REFERENCES}

[1] E. Gaalaas, "Class D Audio Amplifiers: What, Why, and How," in Analog Dialogue, Jun. 2006.

[2] M. Berkhout, "Class D audio amplifiers in mobile applications," in Circuits and Systems, 2009. ISCAS 2009. IEEE International Symposium on, may 2009, pp. $1169-1172$.

[3] R. Cellier, E. Allier, A. Nagari, C. Crippa, R. Bassoli, G. Pillonnet, and N. Abouchi, "A fully differential digital input class D with EMI spreading method for mobile application," in Audio Engineering Society Conference: 37th International Conference: Class D Audio Amplification, Aug 2009. [Online]. Available: http://www.aes.org/e-lib/browse.cfm?elib=15226

[4] M.-L. Yeh, W.-R. Liou, H.-P. Hsieh, and Y.-J. Lin, "An electromagnetic interference (emi) reduced high-efficiency switching power amplifier," Power Electronics, IEEE Transactions on, vol. 25, no. 3, pp. 710-718, 2010.

[5] X. Ming, Z. Chen, Z. kun Zhou, and B. Zhang, "An advanced spread spectrum architecture using pseudorandom modulation to improve emi in class d amplifier," Power Electronics, IEEE Transactions on, vol. 26, no. 2, pp. 638-646, 2011.

[6] Texas Instruments, AN-1497 Filterless Class D Amplifiers, ser. Application Note 1497, May 2006.

[7] Murata, Understanding Ferrite Bead Inductors, Murata, http://www.murata.com/products/emc/knowhow/pdf/23to25e.pdf.

[8] J. Yu, M. T. Tan, S. Cox, and W.-L. Goh, "Time-domain analysis of intermodulation distortion of closed-loop class-D amplifiers," Power Electronics, IEEE Transactions on, vol. 27, no. 5, pp. 2453-2461, May 2012.

[9] F. Koeslag, H. Mouton, and J. Beukes, "Analytical modeling of the effect of nonlinear switching transition curves on harmonic distortion in class D audio amplifiers," Power Electronics, IEEE Transactions on, vol. 28, no. 1, pp. 380-389, Jan 2013.

[10] K. El Haddad, R. Mrad, F. Morel, G. Pillonnet, and A. Nagari, "Ferrite bead effect on Class-D amplifier audio quality," in 17th IEEE Mediterranean Electrotechnical Conference, Apr. 2014, in press.

[11] C. Rostamzadeh, F. Grassi, and F. Kashefi, "Modeling SMT ferrite beads for SPICE simulation," in Electromagnetic Compatibility (EMC), 2011 IEEE International Symposium on, 2011, pp. 530-535.

[12] J. Zhang, K. Qiu, L. Yin, R. Brooks, and B. Chen, "Ferrite bead model extraction and its application in high-performance ASIC analog power filtering," in Electromagnetic Compatibility (EMC), 2011 IEEE International Symposium on, 2011, pp. 536-541.

[13] T. Sato, K. Watanabe, H. Igarashi, T. Matsuo, T. Mifune, K. Kawano, M. Suzuki, Y. Uehara, and A. Furuya, "3-D optimization of ferrite inductor considering hysteresis loss," Magnetics, IEEE Transactions on, vol. 49, no. 5, pp. 2129-2132, 2013.
[14] C. Cuellar, A. Benabou, and N. Idir, "Characterization and modeling of hysteresis for magnetic materials used in emi filters of power converters," Power Electronics, IEEE Transactions on, vol. 29, no. 9, pp. 4911-4920, Sept 2014.

[15] A. Rezaei-Zare, R. Iravani, M. Sanaye-Pasand, H. Mohseni, and S. Farhangi, "An accurate hysteresis model for ferroresonance analysis of a transformer," Power Delivery, IEEE Transactions on, vol. 23, no. 3, pp. 1448-1456, 2008.

[16] S.-T. Liu, S.-R. Huang, and H.-W. Chen, "Using TACS functions within EMTP to set up current-transformer model based on the Jiles-Atherton theory of ferromagnetic hysteresis," Power Delivery, IEEE Transactions on, vol. 22, no. 4, pp. 2222-2227, 2007.

[17] R. Woehrnschimmel, C. Kral, F. Muellner, S. Wild, H. Neudorfer, and F. Dangl, "A combined hysteresis and eddy-current model developed for a wide frequency range in electric machine applications," in Energy Conversion Congress and Exposition (ECCE), 2013 IEEE, 2013, pp. 3180-3185.

[18] L. Cao and G. Li, "Complete parallelogram hysteresis model for electric machines," Energy Conversion, IEEE Transactions on, vol. 25, no. 3, pp. 626-632, 2010.

[19] D. Jiles and D. Atherton, "Theory of ferromagnetic hysteresis (invited)," Journal of Applied Physics, vol. 55, no. 6, pp. 2115-2120, 1984.

[20] P. Wilson, J. Ross, A. Brown, T. Kazmierski, and J. Baranowski, "Efficient mixed-domain behavioural modelling of ferromagnetic hysteresis implemented in VHDL-AMS," in Design, Automation and Test in Europe Conference and Exhibition, 2004. Proceedings, vol. 1, 2004, pp. $742-743$.

[21] A. Raghunathan, Y. Melikhov, J. Snyder, and D. Jiles, "Theoretical model of temperature dependence of hysteresis based on mean field theory," Magnetics, IEEE Transactions on, vol. 46, no. 6, pp. 1507$1510,2010$.

[22] J. Chan, A. Vladimirescu, X.-C. Gao, P. Liebmann, and J. Valainis, "Nonlinear transformer model for circuit simulation," Computer-Aided Design of Integrated Circuits and Systems, IEEE Transactions on, vol. 10, no. 4, pp. 476-482, 1991.

[23] I. Mayergoyz, The Classical Preisach Model of Hysteresis. Springer New York, 1991.

[24] K. Wiesen and S. Charap, "Vector Preisach modeling," Journal of Applied Physics, vol. 61, no. 8, pp. 4019-4021, 1987.

[25] S. Charap and A. Ktena, "Vector Preisach modeling (invited)," Journal of Applied Physics, vol. 73, no. 10, pp. 5818-5823, 1993.

[26] C. Boley and M. Hodgdon, "Model and simulations of hysteresis in magnetic cores," Magnetics, IEEE Transactions on, vol. 25, no. 5, pp. 3922-3924, 1989.

[27] D. Zhang and J. Fletcher, "Quantification of required multi-segments for accurately computing induced voltage in a ferrite inductor using static and dynamic Jiles-Atherton models," Magnetics, IEEE Transactions on, vol. 49, no. 11, pp. 5424-5429, Nov 2013.

[28] A. Benabou, "Contribution à la caractérisation et à la modélisation de matériaux magnétiques en vue d'une implantation dans un code de calcul de champ," 2002, Université de Lille I.

[29] E. D. M. Hernandez, C. S. Muranaka, and J. R. Cardoso, "Identification of the Jiles-Atherton model parameters using random and deterministic searches," Physica B: Condensed Matter, vol. 275 , no. 1-3, pp. $212-215,2000$. [Online]. Available: http: //www.sciencedirect.com/science/article/pii/S0921452699007668

[30] P. Wilson, J. Ross, and A. Brown, "Optimizing the Jiles-Atherton model of hysteresis by a genetic algorithm," Magnetics, IEEE Transactions on, vol. 37 , no. 2, pp. 989-993, 2001.

[31] M. Toman, G. Stumberger, and D. Dolinar, "Parameter identification of the Jiles-Atherton hysteresis model using differential evolution," Magnetics, IEEE Transactions on, vol. 44, no. 6, pp. 1098-1101, 2008.

[32] muRata, BLM18HK102SN1D, Murata Manufacturing Co., Ltd., http://www.murata.com/.

[33] P. Russo, F. Yengui, G. Pillonnet, S. Taupin, and N. Abouchi, "Dynamic voltage scaling for series hybrid amplifiers," Microelectronics Journal, vol. 44, no. 9, pp. 753 - 763, 2013. [Online]. Available: http://www.sciencedirect.com/science/article/pii/S0026269213000864

[34] Bob Metzler, Audio Measurement Handbook, ser. Audio Precision, January 2005.

[35] E. Sturtzer, G. Pillonnet, G. Lemarquand, and N. Abouchi, "Comparison between voltage and current driving methods of a micro-speaker," Applied Acoustics, vol. 73, no. 11, pp. 1087 - 1098, 2012. [Online]. Available: http://www.sciencedirect.com/science/article/ pii/S0003682X12001284

[36] F. Sixdenier, M. Raulet, R. Marion, R. Goyet, G. Clerc, and F. Allab, "Dynamical models for eddy current in ferromagnetic cores introduced 
in an fe-tuned magnetic equivalent circuit of an electromagnetic relay," Magnetics, IEEE Transactions on, vol. 44, no. 6, pp. 866-869, 2008.

[37] T. Chailloux, M. Raulet, C. Martin, C. Joubert, F. Sixdenier, and L. Morel, "Magnetic behavior representation taking into account the temperature of a magnetic nanocrystalline material," Magnetics, IEEE Transactions on, vol. 48, no. 2, pp. 455-458, 2012.

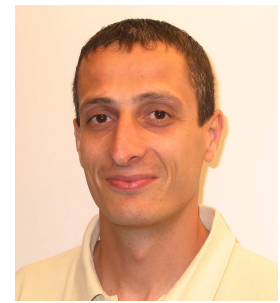

Christian Vollaire was born, in 1968. He received his M.S.c in electrical engineering from the University of Saint Jérôme in Marseille (France) in 1992 and a $\mathrm{PhD}$ degrees in electrical engineering from the Ecole Centrale de Lyon (France) in 1997. From 1997 to 1998 , he worked as graduate research assistant at the Ecole Centrale de Lyon. In 1998, he joined the AMPERE laboratory (UMR CNRS 5005). He carries out its research with AMPERE at the Ecole Centrale de Lyon in the field of the numerical modelling applied to the interaction between electromagnetic field and complex systems. He develops in particular specific formulations and numerical methods for the computation of electromagnetic fields in complex structures. One of the fields of applications relates to electromagnetic compatibility.

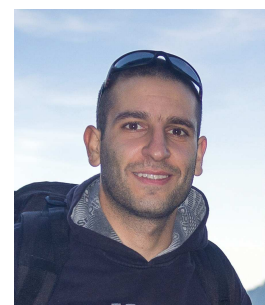

Roberto Mrad was born in Zgharta Lebanon in 1987. He received his master's degree in micro and embedded systems in July 2010 from the Lebanese University. In 2011, he started a PhD with ST-Ericsson and ST Microelectronics (GrenobleFrance), the AMPERE laboratory (Ecole Centrale de Lyon-France) and the INL laboratory (CPE-LyonFrance). The main topic of his research activities is the conducted Electromagnetic Compatibility (EMC) of switching audio amplifier and power converters.

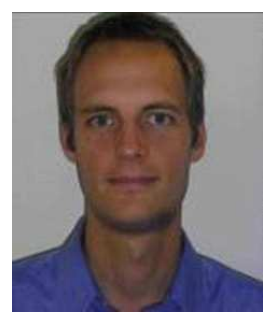

Gael Pillonnet (S'05-M'07) was born in Lyon, France, in 1981. He received his masters degree in Electrical Engineering from CPE Lyon, France in 2004 and $\mathrm{a}$ PhD degree from INSA Lyon, France in 2007. Following an early experience as analog designer in STMicroelectronics in 2008, he joined University of Lyon (CPE Lyon, France) in the Electrical Engineering department. During the 2011-12 academic year, he held a visiting researcher position at the University of California at Berkeley. Since 2013, he has worked as researcher at the CEALETI, a major French research institution. His research focuses on integrated power converters and actuators including modeling, circuit design and control techniques. He has published about 50 papers in his areas of interest, especially in loudspeaker driving.

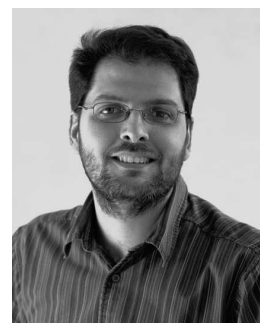

Florent Morel (S'06-M'07) received the Aggregation in electrical engineering from the Ecole Normale Superieure (ENS), Cachan, France, in 2002. Then he received the MSs and Ph.D. diploma in electrical engineering from the Institut National de Sciences Appliquees (INSA), Lyon, France, in 2004 and 2007, respectively. His Ph.D. thesis dealt with predictive control, permanent magnet synchronous machines, voltage source inverters and matrix converters. He is currently with the laboratory AMPERE and the Ecole Centrale, Lyon, France as an associate professor. His research interests include power electronics and electromagnetic compatibility.

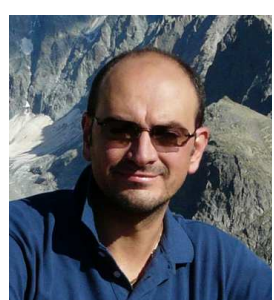

Angelo Nagari Angelo Nagari (IEEE membership: S'92-M'96) was born in Cilavegna, Pavia, Italy, in 1968. He received a degree in electronic engineering (summa cum laude) from the University of Pavia, Italy, in 1993. He was been with STMicroelectronics, Milan, Italy, from 1993, where he worked as a Design Engineer in the analog and mixed IC development for cellular telecommunications. His main research interests were in the fields of Nyquist and Oversampled converters for Systemon-Chip in Audio, RF and Auxiliary applications. In February 2008, he was IP design manager in ST-Ericsson, Grenoble, France. His main role was to define mixed-signal architecture and partitioning for mobile phones platforms and provides IP design on Audio and Power Management fields. In 2011, he was appointed as "Analog \& System IP" Core Competence head for ST-Ericsson analog system development, having as the main objective to lead all related R\& D development. In August 2013, he joined ST-Microelectronics as Design Director of Analog \& Mixed-Signal development center in Grenoble belonging to Analog-MEMS-Sensor group. $\mathrm{He}$ is a reviewer of several IEEE journals (TCAS, JSSC) and permanent member of the ESSCIRC TPC. Within ST-Microelectronics and ST-Ericsson he holds several patents and he gives lectures in many institutes. 